\title{
Le réseau international des Instituts Pasteur : bâtir une science mondiale Olivier Lortholary
}

> Le réseau international des instituts Pasteur représente en 2013 une masse critique de plus de 12000 personnes, répartie sur les 5 continents et fidèle à la triple mission pasteurienne de recherche, formation et action en santé publique dans le domaine des maladies infectieuses. La « trace de l'histoire » est essentielle pour comprendre cette intrication unique de la science et de la médecine, présente dès la création du premier Institut Pasteur à Saïgon (Hô-Chi-Minh-Ville) par Albert Calmette en 1891, soit seulement quatre ans après la fondation de l'Institut Pasteur de Paris, et qui s'établit ensuite aux quatre coins de la planète «pour veiller sur un monde microbien plus turbulent que prévul ». Toujours en Asie, les instituts de Nha Trang (Vietnam) et Hanoï (Vietnam), tous deux maintenant instituts nationaux vietnamiens, furent créés au début du xx siècle, et celui de Phnom Penh en 1953 (il n'a été réouvert qu'en 1995 sous le nom d'Institut du Cambodge, au sortir de plusieurs années de guerre). L'essor du réseau dans cette région du monde, dont le rôle ne cesse de grandir, se traduit par la création de quatre nouveaux instituts : le centre de recherche Université de Hong Kong-Pasteur en 1999, l'Institut Pasteur de Corée du Sud à Séoul en 2004, I'Institut Pasteur de Shanghai-Académie des Sciences de Chine en 2004 [1] $(\rightarrow)$ et, $\rightarrow$ ) Voir l'article de il y a quelques mois, I'Institut Pasteur du de ce numéro Laos (un entretien avec Paul Brey, le directeur de cet institut, sera publié dans cette série). Tous ces instituts au statut très varié se consacrent à la prospection et à la surveillance des populations émergentes d'agents pathogènes et de vecteurs en Asie, notamment virales (citons ici SRAS [syndrome respiratoire aigu sévère], grippe, sida, dengue, hépatite $C$, syndrome pied-main-bouche lié à l'entérovirus $\mathrm{EV} 71$ ), et, en parallèle, à la recherche de thérapeutiques, vaccins et tests diagnostiques innovants.

Si nous avons insisté sur l'aventure asiatique du réseau, c'est parce qu'elle est symbolique tout à la fois de son origine et de son dynamisme actuel, en phase avec l'importance d'une région essentielle d'un point de vue de la recherche en infectiologie. Mais cette série illustrera aussi combien les 24 autres instituts Pasteur ont participé et participent - et pour beaucoup depuis

${ }^{1}$ Citation extraite de l'article de A.M. Moulin : « L'Institut Pasteur au pluriel. Histoire du réseau (2003-2013)». Med Sci (Paris) 2013; 29 (sous presse). le début du Xx $x^{e}$ siècle - en Afrique, Europe, Maghreb-Iran, et aux Amériques [2] $(\rightarrow)$, à l'innovation en infectiologie. Forts de leur histoire dans les domaines épidémiologique, physiopathologique, vaccinal - une des $\rightarrow$ Voir la carte de l'article de Marc Jouan, page 643 de ce numéro caractéristiques de l'histoire du réseau depuis plus de 120 ans -, les instituts ont su développer des technologies sophistiquées, optimiser les stratégies diagnostiques et thérapeutiques pour des maladies tropicales souvent dites négligées, au sein de partenariats multiples avec d'autres instituts de recherche comme avec l'industrie pharmaceutique, mais en respectant l'autonomie et la diversité locales. Il a donc semblé essentiel au comité éditorial de médecine/ sciences et à son rédacteur en chef de donner la parole aux acteurs qui animent aujourd'hui ce réseau à l'Institut Pasteur de Paris et sur le plan international. C'est l'objectif de cette série, faite de onze articles, dont la publication débute aujourd'hui et se poursuivra dans les quatre prochains numéros. Nous avons choisi d'associer dans chacun des numéros des textes scientifiques relatant des réalisations transversales emblématiques des travaux du réseau, et des textes rédigés par ceux qui soutiennent et animent le réseau (comme Chen Zhu [1], Hélène Duchêne [ministère des Affaires étrangères] ou Eliane Coëffier qui présentera les actions de formation du réseau et dont le texte sera accompagné des témoignages de jeunes médecins ou postdoctorants ayant travaillé dans les instituts du réseau). II revient à Marc Jouan, secrétaire général auprès du réseau international, de présenter celui-ci dans sa modernité, faisant face aux défis sanitaires d'aujourd'hui et de demain qui surviennent parfois dans des pays pauvres et instables sur le plan économique et/ou $(\rightarrow)$ Voir l'article de Marc Jouan, page 642 de ce numéro politique [2] $(\rightarrow)$.

L'accord de collaboration récemment signé entre l'OMS (Organisation mondiale de la santé) et le réseau des instituts Pasteur dans les domaines de la réponse aux épidémies et de la sécurité sanitaire mondiale est une reconnaissance de l'intense activité du réseau au service des pays, notamment à ressources limitées, afin de les aider à renforcer leurs capacités en matière d'alerte, de diagnostic et de réponse aux maladies infectieuses. 
Nous avons souhaité que les deux autres articles de ce premier volet de la série soient rédigés par des acteurs éminents de la recherche sur les maladies infectieuses en Asie, lieu de l'émergence par excellence de nombreuses maladies virales.

Le premier, rédigé par Monsieur le Vice-Président de l'Assemblée de la République populaire de Chine, Chen Zhu, ancien ministre de la Santé, présente l'Institut Pasteur de Shanghai-Académie des sciences de Chine (IPS-ASC), créé en 2004 [1] $(\rightarrow)$.

$(\rightarrow)$ Voir l'article de Chen Zhu, page 645 de ce numéro

L'Institut rassemble aujourd'hui plus de 250 scientifiques et est ouvert à l'accueil de jeunes scientifiques français et européens. Le ministre souligne aussi le rôle structurant de l'Institut de Shanghai en matière de formation et son rôle d'acteur de santé publique régional et international, récemment illustré lors de l'épidémie d'infections sévères à entérovirus 71 survenue au Cambodge au cours de l'été 2012. La direction conjointe de I'IPS-ASC par un directeur chinois et un directeur français souligne par ailleurs l'équilibre souhaité entre l'Extrême Orient et le monde occidental, dans un souci de dialogue et de synergie en matière de recherche internationale.

Le second article est rédigé par Didier Ménard (unité d'épidémiologie moléculaire du paludisme à l'Institut Pasteur du Cambodge), Frédéric Ariey et Odile Mercereau-Puijalon (Institut Pasteur, Paris) et porte sur l'étude de la résistance de Plasmodium falciparum aux antipaludiques au sein du réseau international, résistance dont la diffusion a des conséquences désastreuses en santé publique [3] $(\rightarrow)$.

$(\rightarrow)$ Voir l'article de Didier Ménard et al., page 647 de ce numéro

Cet article illustre le décloisonnement actuel de la recherche concernant une des trois priorités du millénaire qu'est le paludisme, qui, par les efforts conjoints des programmes nationaux et internationaux et des unités du réseau paludisme des instituts Pasteur (RIIPPalu), laisse entrevoir des perspectives d'innovation thérapeutique pour enrayer une situation critique à l'échelle mondiale. Les auteurs décrivent concrètement comment les unités du réseau - qui ont une position stratégique et un accès aux technologies de pointe - ont contribué à cette mobilisation : participation aux études multicentriques internationales évaluant actuellement la sensibilité in vitro de $P$. falciparum aux antipaludiques, étude des marqueurs moléculaires impliqués dans la résistance des parasites à divers antipaludiques (notamment séquençage systématique des allèles circulant dans les diverses zones d'endémie), mise au point par l'Institut Pasteur du Cambodge d'un nouveau test in vitro rendant compte de la baisse d'efficacité thérapeutique des artémisinines en Asie.
L'enjeu, en termes de santé internationale, de la résistance de $P$. falciparum aux dérivés des artémisinines survenue en Asie du Sud-Est est en effet crucial. D'autres histoires transversales ayant contribué aux progrès des recherches sur la leishmaniose, la poliomyélite ou l'ulcère de Buruli, seront évoquées dans les prochains numéros.

En conclusion, puissent les lecteurs de médecine/sciences réaliser, à la lecture des onze articles qui forment cette série (dont la publication s'étalera jusqu'en décembre 2013), la contribution majeure du réseau international des instituts Pasteur à l'effort de recherche dans le domaine des maladies infectieuses. Souhaitons aussi que cette série suscite chez de jeunes scientifiques et cliniciens chercheurs le désir de travailler dans un des laboratoires du réseau, à l'occasion d'un stage de maîtrise, doctoral ou postdoctoral, et de découvrir une approche multidisciplinaire de recherche au sein d'une communauté qui poursuit une vraie aventure humaine et scientifique. $\diamond$

Pasteur Institutes, a worldwide network to fight infectious disease

\section{LIENS D'INTÉRÊT}

L'auteur déclare n'avoir aucun lien d'intérêt avec les données publiées dans cet article.

\section{RÉFÉRENCES}

1. Chen Zhu. Quand l'Orient rencontre l'Occident pour une meilleure santé publique. Med Sci Paris 2013 ; 29 : 645-6.

2. Jouan M. Une recherche sur les maladies infectieuses au cœur des zones d'endémie. Med Sci Paris $2013 ; 29: 642-4$.

3. Ménard D, Ariey F, Mercereau-Puijalon 0 . Étude de la résistance de Plasmodium falciparum aux antipaludiques au sein du réseau international des Instituts Pasteur (RIIP-Palu). Med Sci Paris 2013 ; 29 : 647-55.

0 . Lortholary

Institut Pasteur, unité de mycologie moléculaire, Centre national de référence mycoses invasives et antifongiques,

CNRS URA3012,

25, rue du Docteur Roux, 75015 Paris, France et Université Paris-Descartes, hôpital Necker-Enfants malades ; Service des maladies infectieuses et tropicales, APHP, Centre d'infectiologie Necker-Pasteur, Institut Imagine, 149, rue de Sèvres, 75015 Paris, France. olivier.lortholary@pasteur.fr

\section{TIRÉS À PART}

O. Lortholary

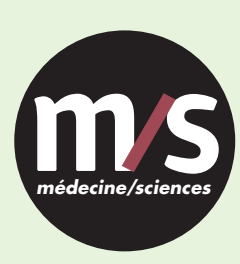

Tarifs d'abonnement $m / s-2013$

$>$ Grâce à $m / s$, vivez en direct les progrès

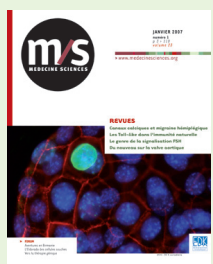

à médecine/sciences

Bulletin d'abonnement page 590 dans ce numéro de $\mathrm{m} / \mathrm{s}$ 